\title{
Construcción y habilitación de la "Bebeteca modelo en Nicaragua" adjunta a la Biblioteca Nacional Rubén Darío
}

\author{
Isolda María Torres, Karla Vanesa Salas Olivares, \\ Gloria Trinidad Romero Aragón.
}

Tutora: Msc. Mey Ling Pérez Cordero

Facultad de Humanidades y Ciencias Jurídicas, Departamento de Historia

\section{RESUMEN}

La presente "Propuesta de construcción y habilitación de una Bebeteca modelo en Nicaragua", pretende exponer la necesidad de la creación y habilitación de una unidad de información especializada en la atención de los más pequeños, se habla de la población en la primera infancia que incluye a niños de 0 - 6 años de vida, lugar donde puedan interactuar con los libros para desarrollar y estimular el hábito lector a través de diversas actividades, logrando un desarrollo integral en nuestra niñez. El edificio será una construcción adecuada, que permita vincular a los bebés, los padres con la lectura, que este sea un lugar de fácil acceso, confortable, cómodos. El Proyecto se iniciará en el mes de mayo 2015, finalizando en el mes de mayo 2020. Estará ubicado en el Distrito II de Managua, en el Barrio Rubén Darío. En la formulación del proyecto se utilizó la metodología del Marco Lógico, para definir el problema, analizar las causas, consecuencias y definir las alternativas de solución. En la definición del problema se tomó en cuenta a todos los involucrados (directos e indirectos) abordándolos a través de encuesta. También se hizo un estudio de mercado, consultando la opinión de los potenciales usuarios a través de una encuesta; ambas muestras se calcularon no probabilísticamente, con un muestreo a conveniencia. Se puede concluir que para mejorar el nivel lector en la población es necesario invertir recursos y tiempo para el fomento de este hábito desde la primera infancia, para lograr que los niños vean la lectura como una necesidad inherente a su desarrollo integral.

Palabras clave: unidad de información, propuesta de habilitación.

\section{SUMMARY}

This "Proposed construction and equipping of model Bebeteca in Nicaragua", aims to expose the need for creating and enabling an information unit specializing in the care of the little ones, there is talk of the town in early childhood including children from 0-6 years, where they can interact with books to promote and encourage reading habits through various activities, achieving comprehensive development in our childhood. The building is an appropriate construction, which allows linking babies with parents in the reading area, so that; this would be an easy, accessible and comfortable place to go. The project will start in May 2015, ending in May 2020. It will be 
located in District II in Managua, in Ruben Dario neighborhood. The logical framework methodology was used in the formulation of the project to define the problem, analyze the causes and consequences to find some solutions. In the definition of the problem, everyone involved (direct and indirect) was taking into account applying them a survey. There was also a market research, consulting the opinion of potential users through a survey; both samples were not probabilistically calculated with a convenience sample. It can be concluded that to improve the reading level in the population is necessary to invest resources and time to promote this habit from infancy, to make children see reading as an inherent to their development needs.

Keywords: information unit, enabling proposal

\section{INTRODUCCIÓN}

El presente estudio es una propuesta de Proyecto de Graduación para optar al título de Licenciadas en "Gestión de la Información" del Departamento de Historia de la Facultad de Humanidades y Ciencias jurídicas de la Universidad Nacional Autónoma de Nicaragua UNANManagua.

Se tiene el interés de presentar esta Propuesta de Proyecto ante las autoridades nacionales correspondientes para que sea tomado en cuenta a la hora de la elaboración de proyectos que contribuyan al desarrollo humano, fortaleciendo sobre todo el hábito lector y por ende al desarrollo cultural de la sociedad.

La primera vez que se escuchó hablar de Bebeteca y su importancia fue en la " $5^{\circ}$ Conferencia Europea de Lectura”, realizada en Salamanca en la Fundación Germán Sánchez Ruipérez, en julio de 1987, se habló ampliamente y por primera vez de los "Servicios de Atención Especial para la Pequeña Infancia (0 a 6 años), conocido internacionalmente como "BEBETECA" (Ana Baeza, 2012, párr. I)

\section{DESARROLLO}

En Nicaragua un alto porcentaje de la población alfabeta no posee un óptimo nivel lector, ésta realidad nos ha encauzado a prestar nuestra atención en la primera infancia con el interés de incidir en el aprendizaje temprano de niños y niñas nicaragüenses, priorizando esta etapa de vida en la que se encuentran las condiciones para cultivar este hábito tan importante, como es la lectura. Sumado a ello, 
existen una serie de políticas y planes de nuestro gobierno en función de atender a los más “chiquitos" y "chiquitas" que crean el clima favorable para iniciativas de esta naturaleza.

Desde la primera infancia e incluso desde que el bebé está en el vientre materno es crucial que se fomente y anime a la lectura, desde esta etapa de su desarrollo es donde se creará el vínculo indestructible entre el bebé y el libro.

Isolda María Torres, Carla Vanessa Salas Olivares y Gloria Trinidad Romero Aragón - estudiantes del último semestre de la Carrera Gestión de la Información del Departamento de Historia de la Universidad Nacional Autónoma de Nicaragua UNAN-Managua del Recinto Universitario "Rubén Darío" -elaboramos una propuesta de Proyecto titulado “Construcción y Habilitación de la Bebeteca Modelo en Nicaragua”.

La idea de este trabajo surge como una inquietud relacionada con nuestro ámbito de competencia en la gestión de la información y la responsabilidad que tenemos en el fomento y animación de la lectura. Las bibliotecas son un eslabón en la formación de futuros lectores, labor que se realiza en Nicaragua a partir de la edad escolar, desatendiendo a la primera infancia o niños entre los 0 y los 6 años de vida, etapa fundamental para desarrollar los buenos hábitos en el bebé, incluyendo desde esta edad el hábito de la lectura.

En la "5a Conferencia Europea de Lectura" celebrada en Salamanca en julio de 1987, en la Fundación Germán Sánchez Ruipérez, se discute de manera significativa el papel e importancia de las Bebetecas, abordando ampliamente distintos aspectos relacionados con los "Servicios de Atención Especial para la Pequeña Infancia (0 a 6 años).

En América Latina existen experiencias relacionadas con el trabajo en unidades de información especializadas para la primera infancia. En Cuba, México y Colombia ya son una realidad, han dado y siguen ofreciendo excelentes resultados, estas experiencias nos alentaron a proponer este proyecto de desarrollo humano y ponerlo en práctica en nuestro país obteniendo los beneficios que traen consigo.

Teniendo conocimiento que las bebetecas son una realidad con excelentes resultados en otros países y los planes y proyectos de nuestro gobierno nacional, expresados en la Política Nacional de Primera Infancia “Amor por los más chiquitos y las más chiquitas (2011)”, que expresa:

La Primera Infancia es un período crucial en la vida del ser humano, por ser la etapa del ciclo de vida en donde se establecen las bases del desarrollo de la persona, la conformación neurológica y neurocognitiva, la estructura ósea, las capacidades de aprendizaje, habilidades y destrezas sensoriales, 
motrices, las relaciones de comunicación e interacción social, los sistemas inmunológicos para evitar las enfermedades, los procesos comunicacionales, emocionales, afectivos; por tanto si se invierte en los más chiquitos y chiquitas estamos garantizando el desarrollo físico, mental, social y espiritual del capital humano del presente y del futuro.

La Neurociencia y la Neuroeducación han demostrado que la inversión en la primera infancia tiene notable éxito en el aprendizaje temprano y durante toda la vida. Esta inversión debe ser oportuna, no se puede esperar hasta que sean adultos, ni siquiera se puede esperar a que lleguen a la edad escolar, podría ser demasiado tarde, potenciar el desarrollo de las funciones biológicas, psicológicas y sociales, a través de la inversión en las niñas y los niños durante sus primeros años, equivale a garantizar futuros provechosos, con bienestar y felicidad.

Los programas sociales del Gobierno dirigidos a la Primera Infancia tendrán efectos duraderos en el aprendizaje y la motivación y la adecuada estimulación temprana, desde la familia, comunidad y la escuela, harán posible en las niñas y niños, el desarrollo pleno de sus estructuras biológicas, psicológicas y sociales.

En Nicaragua se está consciente que no se posee el hábito lector y desde 1981 se han realizado esfuerzos para fomentar y animar este hábito. El 23 de agosto de 1981 se inicia la 1er. Campaña Nacional de Bibliotecas "Carlos Fonseca Amador", que tenía como primer objetivo Incrementar el hábito de lectura en nuestro pueblo. Desde 1989 La Biblioteca Nacional Rubén Darío a través de la Red Nacional de Bibliotecas Públicas, actualmente compuesta por 141 bibliotecas municipales a nivel nacional, promueve y anima la lectura con actividades diversas como Caja Viajera, cuenta cuentos, la hora del cuento, narraciones, concursos de dibujo, concursos de poesía, círculos de lectura, club de lectura, festivales de lectura, maratón de lectura en voz alta, capacitación a niños, jóvenes y adultos para ser promotores de lectura, charlas y exposiciones literarias, exposiciones bibliográficas, plan vacacional de fin de año.

A inicios de los años 90, Mary Jo Amani visita bibliotecas en Nicaragua y observa que carecen de literatura infantil, en 1993 decide crear un organismo que incentive a los niños a leer por lo que funda Libros para Niños (LpN), en el departamento de Rivas. En mayo de 2014 ANIBIPA impulsa y promueve el hábito lector con la Campaña Nacional en Promoción de la Lectura "En Nicaragua todos queremos Leer", con actividades como "La Biblioteca en tu parada", en la parada del Centro Comercial de Linda Vista y en la parada del Zumen, frente al Centro Cívico, en estos lugares se contaron cuentos, se 
distribuyó material informativo de promoción de la lectura y del libro. Además participaron la Universidad Nacional de Ingeniería, Universidad Nacional Agraria, Universidad Nacional Autónoma de Nicaragua,

El Ministerio de Educación (MINED), con el propósito de incentivar el hábito y amor por la lectura, promueve en todos los centros educativos de Educación Primaria, Secundaria y de Formación Docente del país, la Campaña Permanente de Lectura. La campaña de la lectura, tiene diversas estrategias que se promueven en las aulas de clase y en las bibliotecas escolares, como El Club de Lectores, donde se coordina un espacio de lectura en la biblioteca u aula. La Rotación de Lectura, es otra dinámica pedagógica que consiste en que las bibliotecarias se trasladen a las aulas a desarrollar de manera conjunta con los alumnos, la lectura de un cuento, poema o texto.

La restitución de derechos promovida y ejecutada por nuestro Gobierno de Reconstrucción y Unidad Nacional (GRUN), la retomamos y planteamos en nuestra propuesta de proyecto y en la XXXIII Jornada Universitaria de Desarrollo Científico "Roberto González In Memorian. Consideramos es un proyecto pertinente con los lineamientos y políticas del bien común y equidad social, expresados en el Plan Nacional de Desarrollo Humano (2012-2016), de la siguiente manera:

En la búsqueda de mecanismos para revertir el estado de pobreza de los nicaragüenses, a partir del 2007, el Gobierno de Reconciliación y Unidad Nacional ha venido desarrollando una política social desde la restitución de derechos, recuperación de valores y fortalecimiento de capacidades en favor de las familias pobres, generando resultados positivos como la reducción de la pobreza y la desigualdad...En Educación, el Gobierno seguirá garantizando y fortaleciendo el derecho de la población a una educación gratuita, desde un enfoque de derecho humano fundamental, bajo el principio de universalización de la educación para que cada vez más personas, especialmente los más empobrecidos ingresen a las escuelas en la edad que les corresponde y progresen en forma continua...

La construcción y habilitación de una Bebeteca modelo en Nicaragua, se convierte en una realidad tangible en función del avance y fortalecimiento de acciones que contribuyan al desarrollo humano de la sociedad nicaragüense, garantizando de futuro una población con formación integral.

\section{Una unidad de información especializada}

La "Propuesta de construcción y habilitación de una Bebeteca modelo en Nicaragua", plantea la necesidad de creación y habilitación de una unidad de información especializada en la atención de los más pequeños, se habla de la población en la primera infancia que incluye a niños de 0 - 6 años de 
vida, lugar donde puedan interactuar con los libros para desarrollar y estimular el hábito lector a través de diversas actividades, logrando un desarrollo integral en nuestra niñez.

Como resultado de la ejecución de este proyecto se pretende contar con una biblioteca especializada que brinde a la comunidad servicios que hasta la fecha no lo ofrecen las bibliotecas existentes, la bebeteca tendrá como objetivo general "Crear espacios que permitan al niño, sus padres o tutores interactuar e integrarse en las actividades que fomenten el hábito lector, recreando el entorno cultural que la Bebeteca tiene como institución educadora, estimulando el desarrollo afectivo e intelectual y la comunicación entre adultos y niños alrededor del arte y la literatura”.

Entre sus objetivos específicos, destacan: Impulsar el placer por la lectura desde la primera infancia a través de un espacio diseñado para bebés, dotado de bibliografía especializada; Contribuir al fomento, promoción y animación del hábito lector de forma fácil y grata; Establecer un espacio interactivo, seguro, lúdico y creativo para él bebé; así como para el acercamiento del bebé con el mundo de las palabras y las imágenes.

Como Misión se plantea, ofrecer a la población nicaragüense un espacio cálido en donde no sólo se fomente el vínculo afectivo a través de una serie de dinámicas y actividades, sino que haya un acercamiento a la lectura. Su Visión será Incentivar a nivel nacional la creación de espacios que permitan la estimulación y juego para lograr que los más pequeños interactúen con diferentes elementos que estimulen tempranamente todos sus sentidos, abriendo un camino hacia la imaginación, la creatividad y el goce de la lectura en sus diferentes formas".

\section{Importancia del Proyecto}

La construcción y habilitación de la Bebeteca Modelo, contribuirá al fomento y motivación, desde la primera infancia, del hábito lector en la población nicaragüense, además se formará en los padres, tutores y docentes las cimientes para que en su vínculo diario con los niños vean necesario el desarrollo del mismo. Es fundamental que nuestra sociedad vea el hábito lector como un aliado para lograr sus objetivos y metas tanto académicas como profesionales.

El Hábito Lector apoya directamente el fortalecimiento del proceso educativo a través de la mejora del análisis crítico, la buena ortografía y redacción permitiendo el avance económico del país, ya que a un mediano plazo los profesionales y ciudadanía en general tendrán un bagaje cultura mejor que el que se posee en la actualidad, lo que permitirá lograr una mejor calificación de técnicos y profesionales. 


\section{Sus usuarios e impacto}

La Bebeteca tendrá una amplia gama de beneficiarios tanto directos como indirectos; beneficiará de forma directa a la primera infancia, a sus padres o tutores y de forma indirecta a la población en general, niños, adolescentes, jóvenes, adultos y tercera edad de ambos sexos, el sistema educativo.

La ejecución del proyecto traerá varios impactos en la población beneficiada y por ende al país, como es: ofrecer a la población nicaragüense la oportunidad de desarrollar el hábito lector, lo que será motor en su desarrollo intelectual y profesional. Podrá logarse: Un mejor nivel de análisis crítico, mejor rendimiento académico, reducción porcentaje de reprobados escolares y universitarios; Mejor calidad educativa de los estudiantes, profesionales más competitivos y desarrollo económico del país.

\section{CONCLUSIONES}

Para mejorar el nivel lector en la población es necesario invertir recursos y tiempo para el fomento de este hábito desde la primera infancia, para lograr que los niños vean la lectura como una necesidad inherente a su desarrollo integral. Es de vital importancia establecer convenios de coordinación y colaboración con las autoridades municipales a nivel nacional en donde existen bibliotecas públicas municipales, con el propósito de hacer conciencia de la importancia que tiene el crear espacios y habilitarlos para atender a la primera infancia con sus padres y tutores para orientarles en el fomento del hábito lector en sus pequeños.

\section{REFERENCIAS}

Avendaño, Y. (. (s.f.). Bebeteca en la Biblioteca Infantil Paralelepípedo, Santiago de Querétaro, México. Recuperado el 13 de septiembre de 2014, de https://sites.google.com/site/manualdebebeteca/ejemplos-de-bebetecas/bebeteca-en-la-bibliotecainfantil-paralelepipedo-santiago-de-queretaro-mexico

Baeza, A. (enero de 2012). Bebeteca: un rinconcito para los bebés Recuperado el 13 de septiembre de 12014, de bibliogtecarios.es:http://bibliogtecarios.es/anabaeza/bebeteca-un-rinconcito-para-losbebés 
Fuentes, M.B. (s.f). Bibliotecas especializadas: concepto y características. Recuperado el 13 de septiembre de 2014, de 101.net: http://suite101.net/article/bibliotecas-especializadas-concepto-ycaracteristicas-a78842\#.VGVwmTSUfK0

Fundación SM . (s.f.). www.fundacion-sm.org.m Recuperado el 13 de Septiembre de 2014, de www.fundacion-sm.org.m: http://www.fundacion-sm.org.mx/node/49

Gobierno de Reconciliación y Unidad Nacional. (2011). Política Nacional de Primera Infancia Amor por los más chiquitos y chiquitas. Managua, Managua, Nicaragua.

IFLA. (2009). http://www.slideshare.net/. Recuperado el 13 de Septiembre de 2014, de http://www.slideshare.net/: http://www.slideshare.net/alamarque/guía-ifla-de-serviciosbibliotecarios-para-la-primera-infancia

Nicaragua, G. d. (26 de abril de 2000). Decreto 28 / 2000 "Creador de la Boblioteca Nacional Rubén Darío". La Gaceta Diario Oficial , págs. 2094 - 2099. 